\title{
Body mass reduction markedly improves muscle performance and body composition in obese females aged $61-75$ years: comparison between the effects exerted by energy-restricted diet plus moderate aerobic-strength training alone or associated with rGH or nandrolone undecanoate
}

\author{
Alessandro Sartorio ${ }^{1,2}$, Nicola A Maffiuletti ${ }^{2,3}$, Fiorenza Agosti ${ }^{2}$, Pier Giulio Marinone ${ }^{2}$, Saverio Ottolini ${ }^{2}$ \\ and Claudio L Lafortuna ${ }^{4}$ \\ ${ }^{1}$ Division of Metabolic Diseases III, Istituto Auxologico Italiano, IRCCS, Piancavallo (VB), Italy, ${ }^{2}$ Experimental Laboratory for Endocrinological \\ Research, Istituto Auxologico Italiano, IRCCS, Milan, Italy ${ }^{3}$ INSERM/ERIT-M 0207 Laboratory, University of Burgundy, Dijon, France and \\ ${ }^{4}$ Institute of Molecular Bioimaging and Physiology, CNR, Segrate (MI), Italy
}

(Correspondence should be addressed to Alessandro Sartorio, Laboratorio Sperimentale di Ricerche Endocrinologiche (LSRE), Istituto Auxologico Italiano, IRCCS, Via Ariosto 13, 20145 Milano, Italy; Email: sartorio@auxologico.it)

\begin{abstract}
Objective: To investigate the effectiveness of a body mass reduction programme entailing diet caloric restriction and moderate physical activity with or without supplementary treatment with recombinant (r) GH or steroids to improve body composition and muscle performance in severely obese women aged $61-75$ years.

Methods: Twenty women were randomly assigned to one of three groups: body mass reduction alone; body mass reduction plus rGH; body mass reduction plus nandrolone undecanoate. Body composition, isotonic muscle strength and anaerobic power output during jumping were determined before and after the 3-week period.

Results: Whatever the experimental group considered, body mass $(P<0.01)$, body mass index $(P<0.05)$ and fat mass $(P<0.05)$ decreased significantly, whereas muscle strength and power increased significantly $(P<0.05)$ after the intervention.

Conclusion: Small body mass reductions after 3 weeks of energy-restricted diet combined with moderate aerobic and strength exercise are associated with significant improvements in upper and lower limb muscle strength and power and reduction of fat mass in severely obese women aged 61-75 years. Although the association of rGH or nandrolone undecanoate does not appear to exert additional effects on body composition and muscle performance attained by body mass reduction alone, further additional studies with larger study groups, different dosages and more prolonged periods are required for definitive conclusions to be drawn.
\end{abstract}

European Journal of Endocrinology 150 511-515

\section{Introduction}

The beneficial effects of a short-term body mass reduction programme, including diet and combined aerobic and strength training, on body composition and physical performance have recently been demonstrated for severely (grade III) obese young patients (1). The outcome of similar procedures in elderly counterparts is, however, poorly known. Ageing is characterized by sarcopenia (2) - that is, the decline in muscle mass and strength that typically begins at about age 60 years (3) - and by a number of factors, for example decreased production and secretion of growth hormone (GH) (4), that inevitably 'weaken' the elderly individual. This situation is then further aggravated in the case of the aged obese, as a result of the well-known negative effects of adiposity on the release of $\mathrm{GH}$ - a reduction in pulse frequency and decrease in GH half-life (5). A recent study from our laboratory indicated that women older than 50 years who have a high body mass index (BMI; $>40 \mathrm{~kg} / \mathrm{m}^{2}$ ) can be considered to be the most disabled category in motor performance as a consequence of obesity, according to the low anaerobic power output of the lower limb muscles (6).

Since the landmark investigation by Rudman et al. (7), recombinant $(\mathrm{rGH})$ has been administered to healthy elderly individuals to limit the impact of ageing in general. GH treatment has been found to decrease fat mass and increase lean body mass in non-obese elderly 
women, even if these changes were accompanied by several side effects (8) and no improvement in exercise performance (9). However, little is known about the effects of low-dose pharmacological treatment (e.g. rGH or steroids) on the body composition and physical performance of obese elderly women.

This paper aimed at demonstrating whether a 3-week body mass reduction programme, including energyrestricted diet plus moderate aerobic and strength training, combined with rGH or nandrolone undecanoate treatment is more effective than body mass reduction alone in improving body composition and muscle performance in severely obese women aged $61-75$ years.

\section{Subjects and methods}

Twenty obese female volunteers participated in a 3-week body mass reduction programme that included an energy-restricted diet (range: 1100-1500 kcal/day, calculated by subtracting approximately $20 \%$ from individual basal energy expenditure) containing 25\% protein, 25-30\% lipid and 45-50\% carbohydrate, moderate aerobic and strength exercise (five sessions of $35 \mathrm{~min} /$ week), psychological counselling and educational lectures (see (1) for more details). All the patients gave their informed consent to participate in the study, which was approved by the Ethics Committee of the Istituto Auxologico Italiano, and they were randomly assigned to one of the three experimental groups. Eight women participated exclusively in the body mass reduction programme, with no pharmacological treatment (group BMR). Two groups of six patients received nandrolone undecanoate $(80 \mathrm{mg}$ i.m., administered weekly; group BMR + NU) or rGH treatment $(0.1 \mathrm{IU} / \mathrm{kg}$ per week s.c., administered daily; group $\mathrm{BMR}+\mathrm{GH}$ ) during the 3 -week period in addition to the body mass reduction programme.

At baseline, the three groups were matched in terms of age (BMR: 66.5 \pm 4.1 years; BMR $+\mathrm{NU}$ : $64.5 \pm 2.9$ years; BMR + GH: 68.2 \pm 3.3 years; mean \pm s.D.) and body composition (Table 1).

\section{Training}

The typical training session consisted of $30 \mathrm{~min}$ of aerobic activities (10 min on a cycloergometer and $20 \mathrm{~min}$ on a treadmill) performed at $40-60 \%$ of individual maximal oxygen consumption $\left(\mathrm{VO}_{2} \mathrm{max}\right)$, followed by 15 repetitions at $40-60 \%$ of one repetition-maximum (1-RM) load on three isotonic machines (Technogym, Gambettola, Italy).

\section{Testing}

Muscle performance and body composition were assessed before and at the completion of the body mass reduction treatment. Maximum anaerobic alactic power $(\mathrm{W})$ output of the lower limb muscles was calculated during a series of five consecutive jumps by using the sum of the flight and contact time of jumps 2-5 (Optojump, Microgate, Bolzano, Italy), according to the formula proposed by Bosco et al. (10). Maximum strength was quantified as 1-RM load on two isotonic machines (leg- and chest-press). The same machines used during the training sessions were used for the evaluation, thereby retaining specificity between training and testing procedures (i.e. the muscle groups that had been trained were then tested in the same conditions). In addition, isometric bilateral handgrip was also measured with a Jamar dynamometer (Preston Corporation, Jackson, MI, USA) to investigate the effects of the 3-week intervention on muscle groups not specifically trained.

Body composition was determined by means of bioimpedentiometric analysis at frequencies of 1,5 , 10,50 and $100 \mathrm{kHz}$ using a tetrapolar impedance plethysmograph (Human-IM Scan, DS-Medigroup, Milan, Italy) and standardised procedures (11).

Concentrations of insulin-like growth factor (IGF)-I (Nichols Institute, San Juan Capistrano, CA, USA) and

Table 1 Pre- and post-treatment data from the three groups

\begin{tabular}{|c|c|c|c|c|c|c|}
\hline & \multicolumn{2}{|c|}{ Group BMR $(n=8)$} & \multicolumn{2}{|c|}{ Group BMR+ NU $(n=6)$} & \multicolumn{2}{|c|}{ Group BMR + GH $(n=6)$} \\
\hline & Pre & Post & Pre & Post & Pre & Post \\
\hline Body mass (kg) & $100.2 \pm 10.5$ & $95.3 \pm 9.1^{\star * \star}$ & $102.3 \pm 11.9$ & $98.5 \pm 10.7^{\star \star \star \star}$ & $101.1 \pm 4.1$ & $98.1 \pm 4.5^{\star \star}$ \\
\hline $\operatorname{BMI}\left(\mathrm{kg} / \mathrm{m}^{2}\right)$ & $38.9 \pm 2.6$ & $37.4 \pm 2.3^{\star \star \star}$ & $42.3 \pm 3.8$ & $40.9 \pm 3.9^{\star \star \star *}$ & $39.5 \pm 3.0$ & $38.4 \pm 3.3^{*}$ \\
\hline Fat mass $(\%)$ & $53.1 \pm 3.9$ & $50.5 \pm 3.8^{*}$ & $53.5 \pm 7.5$ & $50.9 \pm 7.2^{\star \star}$ & $53.0 \pm 4.6$ & $50.6 \pm 5.0^{\star}$ \\
\hline Fat-free mass $(\mathrm{kg})$ & $46.7 \pm 3.8$ & $47.5 \pm 3.7$ & $47.2 \pm 7.5$ & $48.2 \pm 6.3$ & $47.5 \pm 3.9$ & $48.8 \pm 3.3$ \\
\hline W jumps (W/kg) & $5.0 \pm 1.8$ & $6.0 \pm 2.6^{*}$ & $6.0 \pm 1.6$ & $7.0 \pm 1.0$ ** & $6.0 \pm 2.6$ & $6.8 \pm 2.1^{*}$ \\
\hline 1-RM leg press (kg) & $197.4 \pm 37.9$ & $266.3 \pm 39.8^{\star \star \star}$ & $249.8 \pm 22.7 \dagger$ & $305.5 \pm 58.2^{*}$ & $182.0 \pm 48.5$ & $235.0 \pm 74.7^{\star \star}$ \\
\hline 1-RM chest press (kg) & $26.5 \pm 5.7$ & $36.4 \pm 4.4^{\star \star}$ & $27.4 \pm 6.2$ & $34.5 \pm 5.6^{\star \star \star}$ & $29.2 \pm 8.7$ & $35.3 \pm 8.0^{\star \star \star}$ \\
\hline Handgrip $(\mathrm{kg})$ & $26.1 \pm 5.1$ & $26.9 \pm 4.5$ & $22.5 \pm 3.1 \dagger$ & $23.7 \pm 3.5^{\star} \dagger$ & $29.6 \pm 4.9$ & $31.5 \pm 5.6^{\star}$ \\
\hline IGF-I (ng/ml) & $78.6 \pm 51.1$ & $79.5 \pm 49.6 \dagger+\dagger$ & $89.6 \pm 38.6$ & $105.8 \pm 45.5^{\star} \dagger \dagger$ & $117.8 \pm 28.6$ & $219.6 \pm 57.9^{\star \star \star *}$ \\
\hline
\end{tabular}

Values are mean \pm S.D. BMI, body mass index; BMR, body mass reduction; GH, growth hormone; NU, nandrolone undecanoate; 1-RM, one repetitionmaximum; $W$, anaerobic power.

Significant difference between pre- and post-treatment values: ${ }^{\star} P<0.05,{ }^{* \star} P<0.01$, ${ }^{\star * \star} P<0.001$ (paired Student's $t$-test).

Significantly different from the BMR + GH group: $\dagger P<0.05, \dagger \dagger P<0.01, \dagger^{+}+P<0.001$ (one-way ANOVA followed by Tukey post hoc analysis). 
follicle-stimulating hormone (FSH), luteinising hormone (LH) and testosterone (all from DPC, Los Angeles, CA, USA) were determined using commercial kits, before and at the end of the 3-week period. FSH, LH and testosterone concentrations were determined only in the BMR + NU group. Intra-assay coefficients of variation for these parameters were less than $4 \%$ and interassay coefficients of variation were less than $7 \%$.

\section{Statistical analyses}

For each group, pre- and post-treatment values were compared using a unilateral paired Student's t-test. For all the dependent variables, pre- and post-treatment values and percent changes were compared between the three groups using one-way ANOVA followed by Tukey post hoc analysis. The level of significance was fixed at $P \leq 0.05$ for all procedures.

\section{Results}

Body mass, BMI and fat mass were significantly lower $(P<0.05)$ at the end of the 3 -week intervention, whereas fat-free mass did not change significantly whatever the group (Table 1). Post-treatment changes in body composition were comparable between the three experimental groups (Fig. 1A).

Maximal anaerobic power during jumping and maximal strength assessed on the isotonic machines increased significantly $(P<0.05)$ after training (Table 1), but ANOVA showed no significant difference in percentage gains between the three groups (Fig. 1B). Interestingly, handgrip strength did not change significantly after the 3-week period in the BMR group, but a significant increase $(P<0.05)$ was observed for groups $\mathrm{BMR}+\mathrm{NU}$ and BMR $+\mathrm{GH}$. However, no group effect was observed in relative changes for this variable.

Mean baseline IGF-I concentrations were not significantly different between the three groups (Table 1). No significant changes were found in patients treated with body mass reduction alone $(+3.1 \%)$, whereas IGF-I increased significantly in patients treated additionally with nandrolone $(+19.9 \%, \quad P<0.05)$ and $\mathrm{rGH}$ $(+88.7 \%, P<0.001)$. Post hoc analysis showed that the relative increase for the latter group $(\mathrm{BMR}+\mathrm{GH})$ was significantly greater than that for the two other groups $(P<0.05)$.

Mean FSH, LH and testosterone concentrations were not significantly different in the $\mathrm{BMR}+\mathrm{NU}$ group before (FSH: 34.3 $\pm 16.2 \mathrm{IU} / \mathrm{l}$; LH: $12.4 \pm 7.4 \mathrm{IU} / \mathrm{l}$; testosterone: $57.7 \pm 11.5 \mathrm{ng} / \mathrm{dl}$ ) and after the treatment

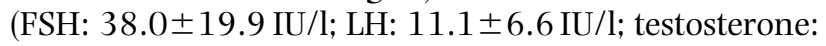
$63.4 \pm 17.3 \mathrm{ng} / \mathrm{dl})$.

No significant side effects were observed during the 3-week period in the three groups.
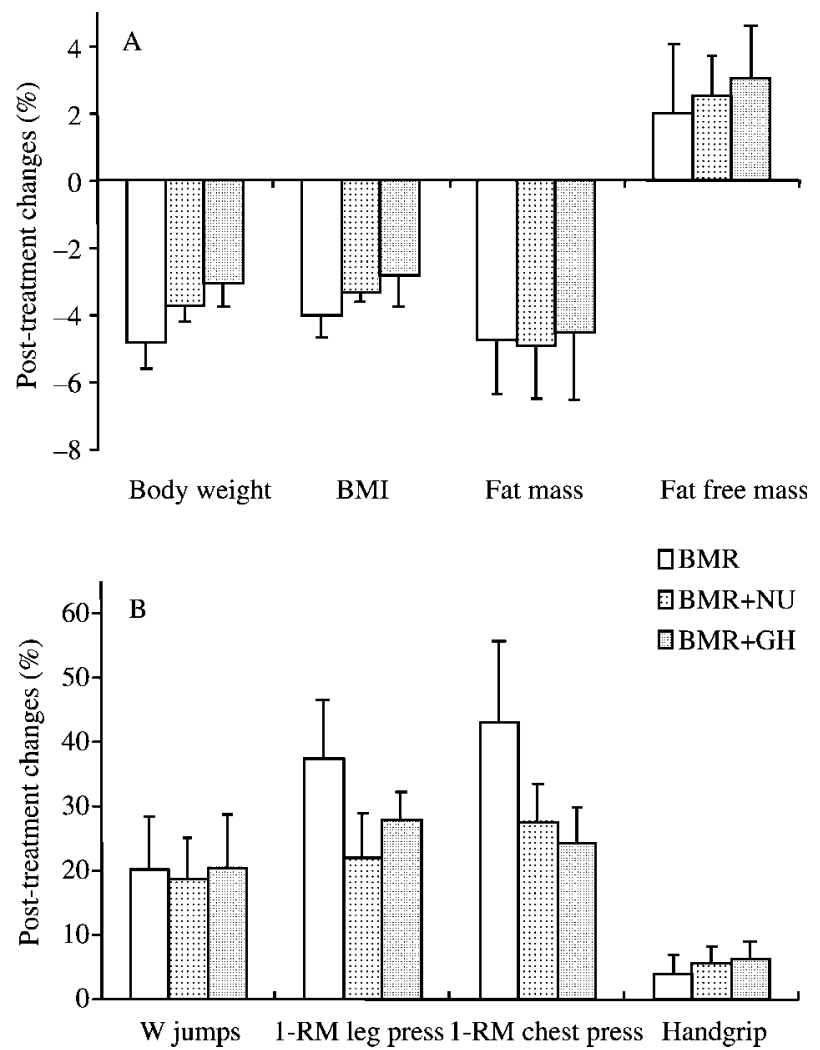

Figure 1 Percent changes between pre- and post-treatment values for the three groups. BMI, body mass index; BMR, body mass reduction; $\mathrm{GH}$, growth hormone; NU, nandrolone undecanoate; 1 -RM, one repetition-maximum; $W$, anaerobic power. Values are mean and S.E.M.

\section{Discussion}

The main finding of this study is that the significant reduction in body mass, BMI and fat mass obtained in the three experimental groups at the completion of the 3-week body mass reduction programme was accompanied by a significant increase in the maximal strength and anaerobic power output of the upper and lower limb muscles, which is likely to improve some activities of daily living in elderly severely obese women. An important finding was that fat-free mass was slightly greater $(2-3 \%)$ in the three experimental groups at the end of the body mass reduction programme, although this was not significant $(P=0.07$ for the two groups treated pharmacologically), explaining at least in part the improvement in muscle strength and power.

Both rGH and nandrolone undecanoate treatments in association with this body mass reduction programme had beneficial effects on body composition and muscle performance comparable to those of body mass reduction alone, suggesting that the observed adaptations could mainly be ascribed to the energyrestricted diet and to the moderate aerobic-strength physical training. However, it is possible that longer 
treatments with greater doses of rGH (and steroids) would exert more evident effects on muscle function than body mass reduction alone, even though they may lead to a greater risk of side effects (8). Bearing in mind the relatively low dosage of nandrolone undecanoate or rGH administered to our patients ( $80 \mathrm{mg} /$ week and $0.1 \mathrm{IU} / \mathrm{kg}$ per week, respectively) and the short duration of the present treatment, we did not observe side effects in severely obese elderly women.

Relative gains in muscle strength after treatment ranged from $22 \%$ to $43.1 \%$, and muscle power during consecutive jumps increased approximately 20\%, in these elderly females. Interestingly, these changes are very similar to those obtained in our laboratory with young obese individuals who participated in the same body mass reduction programme (12). In the same way, changes in body composition obtained here with females aged 61-75 years were comparable to those previously reported for young obese patients (13). Taken as a whole, these results suggest that the adaptability of body and muscle to a 3-week body mass reduction programme is very similar in both elderly and young severely obese patients, as was also noted by Häkkinen et al. (14) to be the case for non-obese individuals. A study is needed to compare accurately the plasticity of body composition and physical performance as a function of age and sex in obese individuals.

The fact that rGH and steroids in association with physical activity and nutritional regimen significantly enhanced the strength of muscular groups not specifically trained (i.e. isometric handgrip), whereas body mass reduction alone had no effect on these groups, is intriguing and requires further investigation, for example in postural muscle groups.

No significant changes in IGF-I concentration were detected in patients treated with energy-restricted diet plus aerobic-strength training, but the addition of rGH was associated with significantly increased IGF-I concentrations $(+88.7 \%)$, although they remained in the normal range for age in all but one of the women. It is of interest that administration of nandrolone undecanoate also led to significantly increased IGF-I concentrations $(+19.9 \%)$, although to a lesser extent. Although this latter finding was somewhat surprising, Gayan-Ramirez et al. (15) have recently reported that the levels of expression of IGF-I mRNA were increased significantly after a training programme combined with low- or high-dose nandrolone decanoate treatment in rat diaphragm.

In conclusion, the addition of $\mathrm{rGH}$ or nandrolone to a 3-week body mass reduction programme combining energy-restricted diet plus moderate aerobic and strength exercise was not more effective than body mass reduction alone in improving body composition and muscle performance in severely obese women aged 61-75 years. Further additional research on a larger study group composed of elderly obese men and women, and for more prolonged periods of observation, is required to confirm these preliminary observations.

\section{Acknowledgements}

This study was partially supported by Progetto di Ricerca Finalizzata 'Fisiopatologia della sarcopenia e sviluppo di strategie multidisciplinari di diagnosi, prevenzione ed intervento sull'anziano', Italian Ministry of Health, Rome, Italy.

The authors acknowledge Dr L Petroni for nutritional counselling and Dr A Miola, Pharmacia Italy, for kindly supplying rGH. The technical assistance of Mr M Proietti was greatly appreciated. The authors are also indebted to Drs G Silvestri, M Resnik and A Tibaldi and to Ms F Pera of the 3rd Division of Metabolic Diseases for their expert assistance during the execution of the tests, and to Dr M Massarini, Technogym Research Centre, for providing testing and training machines.

\section{References}

1 Sartorio A, Lafortuna CL, Massarini M \& Galvani C. Effects of different training protocols on exercise performance during a short-term body weight reduction programme in severely obese patients. Eating and Weight Disorders 20038 36-43.

2 Dutta C, Hadley EC \& Lexell J. Sarcopenia and physical performance in old age: an overview. Muscle and Nerve 19975 (Suppl) S5-S9.

3 Vandervoort AA. Aging of the human neuromuscular system. Muscle and Nerve 200225 17-25.

4 Zadik Z, Chalew SA, McCarter RJ Jr, Meistas M \& Kowarski AA. The influence of age on the 24-hour integrated concentration of growth hormone in normal individuals. Journal of Clinical Endocrinology and Metabolism $198560513-516$.

5 Veldhuis JD, Iranmanesh A, Ho KK, Waters MJ, Johnson ML \& Lizarralde G. Dual defects in pulsatile growth hormone secretion and clearance subserve the hyposomatotropism of obesity in man. Journal of Clinical Endocrinology and Metabolism 199172 51-59.

6 Sartorio A, Proietti M, Marinone PG, Agosti F, Adorni F \& Lafortuna CL. Influence of gender, age and BMI on lower limb muscular power output in a large population of obese men and women. International Journal of Obesity 200428 91-98.

7 Rudman D, Feller AG, Nagraj HS, Gergans GA, Lalitha PY, Goldberg AF et al. Effects of human growth hormone in men over 60 years old. New England Journal of Medicine 1990323 1-6.

8 Thompson JL, Butterfield GE, Marcus R, Hintz RL, Van Loan M, Ghiron L et al. The effects of recombinant human insulin-like growth factor-I and growth hormone on body composition in elderly women. Journal of Clinical Endocrinology and Metabolism $1995 \mathbf{8 0} 1845-1852$.

9 Wideman L, Weltman JY, Hartman ML, Veldhuis JD \& Weltman A. Growth hormone release during acute and chronic aerobic and resistance exercise. Recent findings. Sports Medicine 200232 987-1004.

10 Bosco C, Luhtanen P \& Komi PV. A simple method for measurement of mechanical power in jumping. European Journal of Applied Physiology 198350 273-282.

11 Deurenberg P. International consensus conference on impedance in body composition. Age and Nutrition 19945 142-145.

12 Lafortuna CL, Resnik M, Galvani C \& Sartorio A. Effects of nonspecific vs individualized exercise training protocols on aerobic, anaerobic and strength performance in severely obese subjects 
during a short-term body mass reduction program. Journal of Endocrinological Investigation 200326 197-205.

13 Sartorio A, Ottolini S, Agosti F, Massarini M \& Lafortuna CL. Three-week integrated body weight reduction programme markedly improves performance and work capacity in severely obese patients. Eating and Weight Disorders 20038 107-113.

14 Häkkinen K, Kallinen M, Izquierdo M, Jokelainen K, Lassila H, Mälkiä A et al. Changes in agonist-antagonist EMG, muscle CSA, and force during strength training in middle-aged and older people. Journal of Applied Physiology 199884 1341-1349.
15 Gayan-Ramirez G, Rollier H, Vanderhoydonc F, Verhoeven G, Gosselink R \& Decramer M. Nandrolone decanoate does not enhance training effects but increases IGF-I mRNA in rat diaphragm. Journal of Applied Physiology $2000 \mathbf{8 8}$ 26-34.

Received 3 October 2003

Accepted 9 January 2004 